\title{
Space-Time-Frequency (STF) Coding for MIMO-OFDM Systems
}

\author{
Andreas F. Molisch, Senior Member, IEEE, Moe Z. Win, Senior Member, IEEE, and Jack H. Winters, Fellow, IEEE
}

\begin{abstract}
We consider the capacity of multiple-input-multiple-output (MIMO) systems that use OFDM as the modulation format. We point out a basic equivalence between antennas and $O F D M$-tones. This similarity immediately allows us to essentially reuse all space-time codes designed for flat-fading channels in MIMO-OFDM systems operating in frequency-selective channels. An optimum code would thus code across all antennas and tones (as well as time) simultaneously. Since this can become very complex, we propose a method for grouping antennas and codes in such a way that the inherent diversity is retained, while the complexity is greatly reduced. Capacity computations between the full-complexity and the reduced-complexity systems illustrate this tradeoff.
\end{abstract}

Index Terms-MIMO, OFDM, space-time coding.

\section{INTRODUCTION}

W IRELESS SYSTEMS with multiple antennas at the transmitter and receiver (multiple-input-multiple-output, MIMO) have much larger capacity in fading channels than standard wireless systems [1], [2]. The appropriate use of space-time (ST) processing [3] and ST codes [4] allows us to achieve, or at least approach, these capacities in practical systems. For frequency-selective channels, a combination of MIMO with OFDM (orthogonal frequency division multiplexing) is promising [5], [6]. The simplest way to perform ST coding in a MIMO-OFDM system would be to apply the ST-codes for the frequency-flat channels to each tone separately. However, a recent paper [7] has pointed out that this is suboptimum, as the inherent frequency diversity of the frequency-selective channel is not exploited. It was also stated that construction of codes that code across tones would be difficult. In this paper, we show by a very simple observation how we can extend ST code design rules to frequency selective channels without sacrificing performance. We will also develop further simplifications and illustrate the performance-complexity tradeoff.

\section{Antennas And Tones-Unified Perspective}

In a conventional OFDM system [see Fig. 1(a)], i.e., without exploitation of the frequency diversity, the data streams for the OFDM tones enter separate ST coders whose outputs are then forwarded to the different antennas. The tones at each antenna are inverse Fourier-transformed and the resulting time signal is

Manuscript received October 12, 2001. The associate editor coordinating the review of this letter and approving it for publication was Dr. J. Ritcey.

The authors were with the Wireless Systems Research Department, AT\&T Labs-Research, Middletown, NJ 07748-4801 USA (e-mail: Andreas.Molisch@ieee.org; win@ieee.org; jack.winters@ieee.org).

Publisher Item Identifier 10.1109/LCOMM.2002.802047.

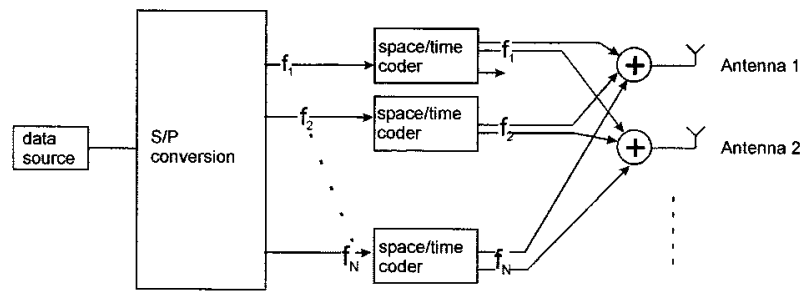

(a)

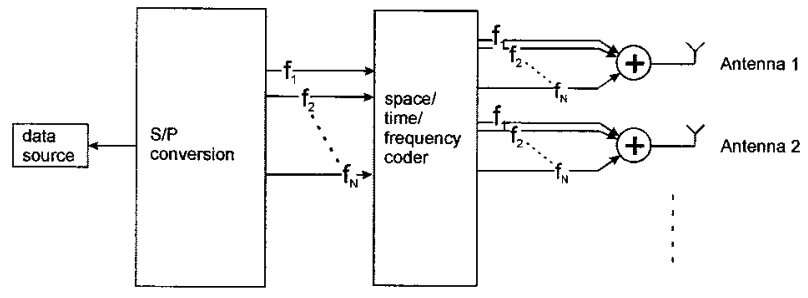

(b)

Fig. 1. System model for: (a) separate coding for each tone (upper figure) and (b) joint space/time/frequence coding (lower figure). IFFT blocks not shown for simplicity.

upconverted to the carrier frequency and transmitted across the mobile radio channel.

As pointed out in [7], coding across the tones is required to exploit the inherent frequency diversity in a time-dispersive channel. A full-complexity coder thus must use the symbols from all tones as input, and distribute them to all tones on all antennas jointly [Fig. 1(b)]. Assuming $N_{\mathrm{t}}$ transmit antennas and $N_{\mathrm{f}}$ tones, the size of the coder is thus $N_{\mathrm{t}} N_{\mathrm{f}} \cdot{ }^{1}$ Systematic methods for designing these codes thus seem difficult to derive. ${ }^{2}$ From the antennas, the signal is sent through the mobile radio channel, which is assumed to be constant within one OFDM block. The fading of the signals at the different antenna elements is assumed to be identically distributed, but not necessarily independent [9], [10]. The channel matrix can thus be written as

$$
\widehat{H}(f)=\left(\begin{array}{ccc}
h_{11}(f) & \ldots & h_{1 N_{\mathrm{t}}}(f) \\
\ldots & \ldots & \ldots \\
h_{N_{\mathrm{r}} 1}(f) & \ldots & h_{N_{\mathrm{r}} N_{\mathrm{t}}}(f)
\end{array}\right)
$$

where $h_{i j}(f)$ denotes the transfer function at frequency $f$ between the antenna pair $(i, j)$. The $h_{i j}(f)$ are zero-mean,

\footnotetext{
${ }^{1}$ In a slight abuse of notation, we call the number of outputs of a (vector) coder the "size" of the coder. The total number of possible signal constellations

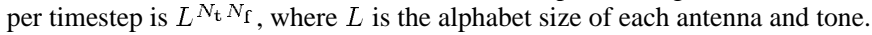

${ }^{2}$ We note that existing system proposals use a rudimentary form of coding across the tones. Any standard coder (e.g., Reed-Solomon code) that is applied to the data stream before serial/parallel conversion distributes the redundancy across tones and thus exploits frequency diversity in some way [8]. However, it seems difficult to formulate analytical criteria and systematic construction methods for ST codes from that point of view.
} 
circularly symmetric complex Gaussian random variables. The channel also adds white Gaussian noise, which is assumed to be independent at all $N_{\mathrm{r}}$ receiver antenna elements.

Let us now consider the similarity between antennas and tones. ${ }^{3}$ We will start out with the simplest imaginable system, to illustrate the basic similarity between tones and antennas, and then step by step relax the assumptions and show where the practical differences lie.

Let us first consider an FDM system with tones that have a narrow bandwidth, yet are widely spaced apart. Similarly, the antennas are assumed to be spaced wide enough apart to exhibit independent fading. Finally, we will assume that the $i$-th transmit antenna can only communicate with the $i$-th receive antenna, so that there is no crosstalk between the antennas. Thus, we have $N_{\mathrm{t}} N_{\mathrm{f}}$ parallel (isolated) communications channels. It does not matter whether they are distinguished by different tones or different antennas-in any case, they are independently fading, and experience no crosstalk. Coding across all available channels obviously allows us to essentially eliminate the capacity fluctuations due to fading, by exploiting the inherent diversity among tones and antennas, and thus achieve outage capacities that are close to the mean capacities.

In the next step, let us relax the condition that there must not be crosstalk between the antennas and between the tones: for different antennas, crosstalk always occurs, while crosstalk across tones is not likely. In particular, there is no crosstalk between widely separated tones. Even if tones are overlapping, as occurs in OFDM, crosstalk is eliminated by the carrier orthogonality and, additionally in time-dispersive environments, by the use of a cyclic prefix. Thus, this is one point where the antennas and the tones do not have a full analogy. However, such an analogy can be recovered by two considerations.

1) Viewing the antennas as additional tones: If the channel is known, a singular value decomposition of the channel matrix transforms it into isolated (but not identically distributed) channels. This recovers analogy to the isolated tones. Note that the transformation from the antenna space to the singular vector space is linear.

2) Viewing the tones as additional antennas: In this case, the "additional antennas" just exhibit a special type of crosstalk description (namely, zero crosstalk). Note that the crosstalk might be beneficial for diversity purposes. However, crosstalk between tones could be enforced, e.g., by using multicarrier-CDMA instead of conventional OFDM. ${ }^{4}$

From these considerations it follows that, in principle, any ST code that is devised for a flat-fading channel can be used in a frequency selective channel. The size of the $\mathrm{ST} \operatorname{code}^{5}$ is now determined by the product of the number of antennas and the number of tones. Thus, codes with a much larger size than those for flat-fading MIMO systems are required. Nonetheless, the code construction rules and evaluation methods remain valid;

\footnotetext{
${ }^{3}$ We stress that the analogy between antennas and tones is only valid from the viewpoint of diversity. In contrast to tones, antennas are also used to provide antenna gain and interference supression.

${ }^{4}$ By similar considerations, one could derive, e.g., an analogy between multiple antennas and orthogonal ST block coding, where $H$ then has a special (unitary) structure. Other analogies, e.g., with SISO block coding, are possible as well.

${ }^{5}$ We are using "size" of the code in the same way that we define the size of the coder.
}

furthermore, code designs for large constellation sizes have recently been hinted at [11].

Summarizing, the transfer function of the MIMO-OFDM system can be written as

$$
\underline{H}=\left(\begin{array}{ccc}
\underline{G}_{11} & \cdots & \underline{G}_{1 N_{\mathrm{t}}} \\
\cdots & \cdots & \cdots \\
\underline{G}_{N_{r} 1} & & \underline{G}_{N_{\mathrm{r}} N_{\mathrm{t}}}
\end{array}\right)
$$

where each entry block

$$
\underline{G}_{i j}=\left(\begin{array}{ccc}
H_{i j}\left(f_{1} \rightarrow f_{1}\right) & \ldots & H_{i j}\left(f_{1} \rightarrow f_{N_{\mathrm{f}}}\right) \\
\cdots & \ldots & \cdots \\
H_{i j}\left(f_{N_{\mathrm{f}}} \rightarrow f_{1}\right) & \ldots & H_{i j}\left(f_{N_{\mathrm{f}}} \rightarrow f_{N_{\mathrm{f}}}\right)
\end{array}\right)
$$

is itself a matrix. If a cyclic prefix of sufficient duration (longer than the maximum excess delay) is used, and no intercarrier interference occurs, $\underline{G}_{i j}$ becomes a diagonal matrix. The correlation between the elements of $G_{i j}$ in (3) depends on the frequency correlation function of the channel. Still, in principle, all entries $H_{i j}\left(f_{k} \rightarrow f_{m}\right)$ (distinguished by antennas $i, j$ and/or tones $k, m$ ) of $H$ are equivalent in the sense that they make up the total matrix, and thus contribute to the capacity.

Also note that coding in time is possible, thus giving diversity in three dimensions, namely, space, frequency, and time. The coding in time is completely identical to the time-component of any ST code for flat-fading channels. The "coding across tones" is more effective in situations where little time diversity can be achieved. If ample time diversity is available, then full capacity can be achieved on each tone, and additional frequency diversity does not have a large advantage. However, in many practical cases, ample time diversity is not available, as either TX and RX are stationary (wireless local loop systems, nomadic mobility), and/or long delays (meaning longer than several coherence times) are not allowed for the coder.

\section{REDUCED-COMPLEXITY SYSTEMS}

Equations (2) and (3) show that a full-complexity system requires a coder of size $N_{\mathrm{t}} N_{\mathrm{f}}$. We thus propose in the following to exploit the typical properties of an OFDM system to reduce the complexity.

The advantage of coding across the tones comes from the exploitation of the frequency diversity in a delay-dispersive channel. Due to restrictions on the duration of the cyclic prefix, the spacing between the tones is usually much smaller than the coherence bandwidth. Distributing information between two neighboring tones thus will not enhance the system's robustness against fading. Rather, only the distribution of information into widely separated tones will contribute to system improvement. As a rule of thumb, the required separation is on the order of one coherence bandwidth.

We thus suggest to perform coding only across tones that are separated by approximately the coherence bandwidth, i.e., to code across a group of tones (see Fig. 2) that is defined as $k=m+n N_{\text {spacing, }}, n=0, \ldots,\left(N_{\mathrm{t}} / N_{\text {spacing }}\right)-1$ for each $m=1, \ldots, N_{\text {spacing. }}$ Thus, the information is distributed onto parallel carriers that are "almost"independently fading, resulting in a high degree of diversity. 


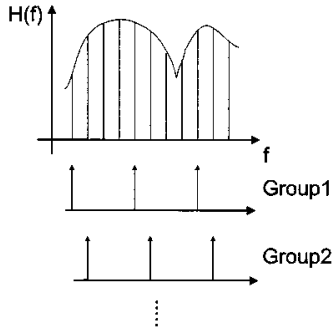

Fig. 2. Grouping frequencies for complexity reduction.

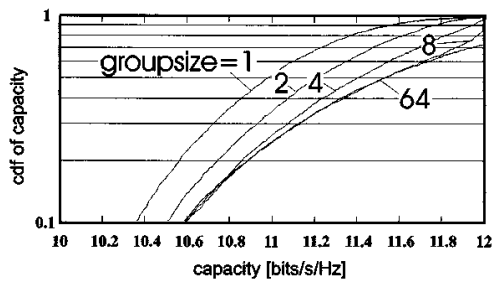

Fig. 3. Cdf of the capacity in a $2 * 2$ OFDM MIMO system with 64-QAM. Lorentzian correlation function with coherence $\mathrm{BW}=$ eight tone distances.

\section{RESULTS}

The capacity of a MIMO-OFDM system coding across the tones is given by

$$
\begin{aligned}
C & =\log _{2}\left[\operatorname{det}\left(I_{N_{\mathrm{r}} N_{\mathrm{f}} \times N_{\mathrm{r}} N_{\mathrm{f}}}+\frac{\bar{\Gamma}}{N_{\mathrm{t}} N_{\mathrm{f}}} \underline{H H}^{H}\right)\right] \\
& =\sum_{i} \log _{2}\left[\operatorname{det}\left(I_{N_{r} \times N_{r}}+\frac{\bar{\Gamma}}{N_{\mathrm{t}} N_{\mathrm{f}}} \underline{\widehat{H}}\left(f_{i}\right) \underline{\hat{H}}\left(f_{i}\right)^{H}\right)\right]
\end{aligned}
$$

where $I_{N_{\mathrm{r}} \times N_{\mathrm{r}}}$ is the $N_{\mathrm{r}} \times N_{\mathrm{r}}$ identity matrix and $\bar{\Gamma}$ is the mean signal-to-noise ratio (SNR) per receiver branch. The above formulation assumes that no time diversity is exploited. It would be easy to include that also, but would add no new insights. Note that $\underline{\hat{H}}\left(f_{i}\right)$ and $\underline{\hat{H}}\left(f_{j}\right)$ exhibit correlation. A simulation of the capacity just requires generation of (correlated) channel matrices, and numerical evaluation of (4).

For the reduced-complexity system, each group $\mathcal{G}_{m}$ of tones of size $N_{G}$ provides capacity (which can be approached by an ideal code)

$$
C_{m}=\sum_{i \in \mathcal{G}_{m}} \log _{2}\left[\operatorname{det}\left(I_{N_{\mathrm{r}} \times N_{\mathrm{r}}}+\frac{\bar{\Gamma}}{N_{\mathrm{t}} N_{\mathrm{f}}} \underline{\widehat{H}}\left(f_{i}\right) \underline{\hat{H}}\left(f_{i}\right)^{H}\right)\right]
$$

and the total capacity is the sum of the capacities of all groups.

In real-world systems, the maximum capacity per tone is limited by the size of the modulation alphabet, e.g., to $C_{\mathrm{QAM}}=$ $6 \mathrm{bits} / \mathrm{s} / \mathrm{Hz}$ for $64-\mathrm{QAM}$. The capacity for coding across groups of size $N_{G}$ is then

$$
C=\sum_{\mathcal{G}_{m}} \min \left(C_{m}, N_{t} N_{G} C_{\mathrm{QAM}}\right) .
$$

Fig. 3 shows the capacity of a system with 64 tones and a coherence bandwidth of 8 (measured in units of tone spacings). The frequency correlation function is assumed to be Lorentzian. The SNR is $20 \mathrm{~dB}$ and the capacity results are normalized to the bandwidth. We see that using ST coders of size 4 (2 antennas and 2 tones) the loss in $10 \%$-outage capacity is less than 0.1 $\mathrm{bit} / \mathrm{s} / \mathrm{Hz}$, while coding on each tone separately results in a loss of $0.2 \mathrm{bits} / \mathrm{s} / \mathrm{Hz}$. When the separation between adjacent tones in one group is equal to the coherence width, the loss in outage capacity (compared to the coding across all 64 tones) is negligible. We have also analyzed situations with a smaller coherence bandwidth. It is intuitively clear that the gain by coding across the tones is larger in that case, but that $N_{G}$ must be larger to fully exploit that possibility. Specifically, with a coherence bandwidth of 2 tone spacings, the loss in outage capacity is $0.45,0.2,0.1$, and 0.02 for $N_{G}=1,2,4$, and 8 , respectively.

\section{SUMmary AND CONCLUSIONS}

We have investigated STF codes for MIMO-OFDM. Starting from the premise that coding across the tones must be done in a systematic way, we have pointed out the basic mathematical analogy between antennas (or spatial eigenmodes) and tones, and explained how this similarity allows to reuse the concepts of ST coding for space-time-frequency (STF) coding required for OFDM. We then proposed a reduced-complexity scheme that codes only across tones that are separated by about one coherence bandwidth. A logical next step would be to use real-world codes on that scheme and investigate performance with full- and reduced-complexity schemes.

\section{ACKNOWLEDGMENT}

The authors thank Dr. L. Cimini, Dr. S. Mueller-Weinfurtner, and Dr. Y.-S. Choi for helpful discussions and critical reading of the manuscript. They also thank the anonymous reviewers for helpful suggestions, especially for pointing out the analogy between antennas and block codes.

\section{REFERENCES}

[1] J. H. Winters, "On the capacity of radio communications systems with diversity in Rayleigh fading environments," IEEE J. Select. Areas Commun., vol. 5, pp. 871-878, June 1987.

[2] G. J. Foschini and M. J. Gans, "On limits of wireless communications in fading environments when using multiple antennas," Wireless Pers. Commun., vol. 6, pp. 311-335, 1998.

[3] G. J. Foschini, "Layered space-time architecture for wireless communication in a fading environment when using multi-element antennas," Bell Labs Tech. J., no. Autumn, pp. 41-59, 1996.

[4] V. Tarokh, N. Seshadri, and A. R. Calderbank, "Space-time codes for high data rate wireless communication: Performance criterion and code construction," IEEE Trans. Inform. Theory, vol. 44, pp. 744-765, 1998.

[5] Y. Li, N. Seshadri, and S. Ariyavisitakul, "Channel estimation for OFDM systems with transmitter diversity in mobile wireless channels," IEEE J. Select. Areas Commun., vol. 17, pp. 461-471, 1999.

[6] H. Boelcskei, D. Gesbert, and A. Paulraj, "On the capacity of wireless systems employing OFDM-based spatial multiplexing," IEEE Trans. Commun., vol. 50, pp. 225-234, 2002.

[7] H. Boelcskei and A. J. Paulraj, "Space-frequency coded broadband OFDM systems," in Proc. IEEE Wireless Commun. Network Conf., 2000, pp. 1-6.

[8] D. Agrawal, V. Tarokh, A. Naguib, and N. Attached are the proofs for your article, CL 2002-249, scheduled to be in the September 2002 issue of IEEE Communications Letters.Seshadri, "Space-time coded OFDM for high data-rate wireless communication over wideband channels," in Proc. VTC'98, vol. 3, 1998, pp. 2232-2236.

[9] A. F. Molisch, M. Steinbauer, M. Toeltsch, E. Bonek, and R. Thoma, "Capacity of MIMO systems based on measured wireless channels," IEEE J. Select. Areas Commun., vol. 20, pp. 561-569, 2002.

[10] D. Gesbert, H. Boelcskei, and A. Paulraj, "Outdoor MIMO wireless channels: Models and performance prediction," IEEE Trans. Commun., to be published.

[11] R. Blum, Q. Yao, Y. Li, and J. H. Winters, "Improved technqiues for 4 transmit and 4 receive antenna MIMO-OFDM for wireless communications," in Proc. 53rd IEEE Vehicular Techn. Conf., 2001, pp. 1298-1302. 